\title{
The role of CSDE1 in translational reprogramming and human diseases
}

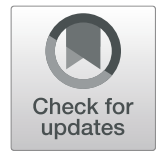

Ao-Xiang Guo ${ }^{1,2,3,4}$, Jia-Jia Cui 1,2,3,4, Lei-Yun Wang ${ }^{1,2,3,4}$ and Ji-Ye Yin ${ }^{1,2,3,4,5,6^{*}}$

\begin{abstract}
CSDE1 (cold shock domain containing E1) plays a key role in translational reprogramming, which determines the fate of a number of RNAs during biological processes. Interestingly, the role of CSDE1 is bidirectional. It not only promotes and represses the translation of RNAs but also increases and decreases the abundance of RNAs. However, the mechanisms underlying this phenomenon are still unknown. In this review, we propose a "protein-RNA connector" model to explain this bidirectional role and depict its three versions: sequential connection, mutual connection and facilitating connection. As described in this molecular model, CSDE1 binds to RNAs and cooperates with other protein regulators. CSDE1 connects with different RNAs and their regulators for different purposes. The triple complex of CSDE1, a regulator and an RNA reprograms translation in different directions for each transcript. Meanwhile, a number of recent studies have found important roles for CSDE1 in human diseases. This model will help us to understand the role of CSDE1 in translational reprogramming and human diseases.
\end{abstract}

Keywords: CSDE1, UNR, Reprogramming, Translation initiation, RNA binding protein, Cancer

\section{Background}

Gene expression is regulated at two major levels: transcription and translation [1]. Translation is the process of transforming intracellular mRNA into protein, and translational homeostasis is critical for cells to properly perform their functions [2]. When the cellular internal or external environment is changed, the translation of a subset of mRNAs is reprogrammed to determine cell fate [35]. As the final step in gene expression, translation is more directly and quickly regulated compared with other steps. Thus, translational reprogramming is critical for cells to rapidly adapt to environmental changes.

Evidence indicates that CSDE1, also known as upstream of N-RAS (UNR), plays an important role in translational reprogramming. It is an RNA-binding protein (RBP) that contains five cold shock domains and is mainly expressed in the cytoplasm [6-9]. CSDE1 plays an important role in a wide range of biological processes, including the cell cycle [10], apoptosis [11], differentiation [12] and dosage

\footnotetext{
* Correspondence: yinjiye@csu.edu.cn

'Department of Clinical Pharmacology, Xiangya Hospital, Central South University, Changsha 410078, People's Republic of China

${ }^{2}$ Institute of Clinical Pharmacology, Central South University; Hunan Key Laboratory of Pharmacogenetics, Changsha 410078, People's Republic of China

Full list of author information is available at the end of the article
}

compensation [13] (Table 1). CSDE1 controls translation initiation of some mRNAs and affects their expression. Furthermore, CSDE1 determines the fate of mRNAs by changing their stability and abundance [26]. Thus, it is a critical factor during the translational reprogramming process. Furthermore, CSDE1 plays a dual role in regulating translation and the abundance of RNAs. However, the mechanisms underlying this phenomenon are still unknown.

In this review, we focus on the bidirectional reprogramming functions of CSDE1 and propose a theoretical model to explain the underlying mechanism. This mechanism may contribute to understanding the role of CSDE1 in human diseases. CSDE1 structure, expression, activity and regulation have been discussed in previous publications $[27,28]$. They will not be discussed in this review.

\section{Bidirectional translational reprogramming Promoting and repressing cap-independent translation initiation}

For translation control, the initiation stage is the ratelimiting step [29]. In eukaryotes, most translation initiation events rely on binding of the cap-binding complex at the 5 ' end of mRNA [30,31]. However, under the conditions of impaired canonical cap-dependent translation, 
Table 1 Reprogramming role of CSDE1 in biological processes and diseases

\begin{tabular}{|c|c|c|}
\hline Biological processes and diseases & The role of CSDE1 & References \\
\hline \multicolumn{3}{|l|}{ Biological processes } \\
\hline Cell cycle & Promotion & [10] \\
\hline Apoptosis & Promotion & [11] \\
\hline Differentiation & Repression & [12] \\
\hline Dosage compensation (male) & Promote DCC assembly & [14] \\
\hline Dosage compensation (female) & Repress DCC formation & [13] \\
\hline \multicolumn{3}{|l|}{ Tumors } \\
\hline Melanoma & Oncogene & [15] \\
\hline Colorectal cancer & Oncogene & (98) \\
\hline Glioma & Oncogene & [16] \\
\hline Breast cancer & Oncogene & [17] \\
\hline PCCS \&PGLS & Tumor suppressor & [18] \\
\hline Oral squamous cell carcinoma & Tumor suppressor & [19] \\
\hline Pancreatic ductal adenocarcinoma & Prognostic biomarker & [20] \\
\hline Epithelial ovarian cancer & Platinum resistance gene & [21] \\
\hline \multicolumn{3}{|l|}{ Other diseases } \\
\hline Autism spectrum disorder & Loss of function mutation & [22] \\
\hline Diamond-Blackfan anemia & Low expression & [23] \\
\hline Embryonic development & Prevent ESC differentiation & {$[24,25]$} \\
\hline
\end{tabular}

cap-independent translation is required for cell survival and stress recovery [32-34]. During this process, $40 \mathrm{~S}$ ribosomes can be directly recruited via an internal ribosome entry site (IRES) element to the 5' untranslated region (UTR) of mRNA [35, 36]. IRES trans-acting factors (ITAFs) are necessary for helping to recruit $40 \mathrm{~S}$ ribosomes to promote translation. CSDE1 is a special ITAF [37]. It is involved in translation regulation mainly via promoting and repressing IRES-mediated cap-independent translation initiation. IRES elements exist in both eukaryotes and viruses [38]. CSDE1 can promote cap-independent mRNA translation initiation via IRES elements in both eukaryotes $[39,40]$ and viruses $[41,42]$.

In eukaryotes, IRES-mediated translation is widely found to be reprogrammed by CSDE1. Apoptosis is the process of programmed cell death. Apoptotic peptidase activating factor 1 (Apaf-1) is a key protein with the capacity to activate caspase 9 during the apoptotic process, and the translation initiation of Apaf- 1 is primarily mediated by its IRES element [43]. The role of CSDE1 in promoting Apaf-1 IRES-mediated translation initiation has been thoroughly studied. In the rabbit reticulocyte lysate (RRL) system, Apaf-1 IRES activity was stimulated by adding purified CSDE1 protein [11]. In cells, Apaf-1 IRES activity was also significantly correlated with the expression level of CSDE1. Transfection of CSDE1 into cell lines (COS7, MRC5, SY5Y and BALB/c) increased Apaf-1 IRES activity by approximately 2.5 -fold. However, transfection of CSDE1 into HeLa and HEK293 cells had no additional stimulation of IRES-independent translation [11], which could suggest that the translation stimulation of CSDE1 on Apaf-1 IRES is cell type specific. In addition, CSDE1 could bind to the Apaf-1 IRES to change its structure and permit the binding of other ITAFs. Through this mechanism, CSDE1 could further stimulate Apaf-1 IRES-dependent translation with the help of other protein regulators [11].

The cell cycle is the most important process for cell growth and proliferation. $\mathrm{p} 58^{\mathrm{PITSLRE}}$ is a PITSLRE protein kinase isoform that is essential for the cell cycle [44]. The translation of P58 ${ }^{\text {PITSLRE }}$ was mediated by an IRES element in the PITSLRE mRNA. In HEK293T cells, CSDE1 barely bound to the PITSLRE IRES element, and there was nearly no expression of $\mathrm{p} 58^{\text {PITSLRE }}$ in the G1 phase [40]. However, during the G2/M phase, when global translation (especially cap-dependent translation) was repressed, p58 ${ }^{\text {PITSLRE }}$ expression was greatly enhanced due to the significant increased binding of CSDE1 on its IRES element [10, 40]. Deletion of CSDE1 binding sites from the human PITSLRE CDNA decreased IRES activity by nearly 50\% [40]. In addition, CSDE1 showed a positive effect on PITSLRE IRES activity in an RRL system [40]. Therefore, CSDE1 is necessary for PITSLRE IRES-dependent translational reprogramming during the cell cycle.

During tumourigenesis, c-myc mRNA is an oncogene involved in cell growth and death. It was reported that c- 
myc overexpression in various cells was mainly due to the aberrant activation of its IRES in the 5'UTR [45, 46]. CSDE1 could bind to the c-myc IRES element and have little or no effect on its activity in RRL [11]. However, CSDE1 greatly increased c-myc IRES activity in both RRL and HeLa cells by working together with other ITAFs, including poly $(\mathrm{rC})$ binding protein 1 (PCBP1), poly $(\mathrm{rC})$ binding protein 2 (PCBP2), heterogeneous nuclear ribonucleoprotein $\mathrm{K}$ (hnRNPK) and UNR-interacting protein (Unrip) [47]. Thus, CSDE1 is a positive regulator of c-myc IRES activity.

For viruses, CSDE1 also stimulates cap-independent translation initiation. Human rhinovirus (HRV) and poliovirus (PV) mainly utilize IRES-driven translation to promote their protein synthesis [41]. CSDE1 plays an important role in the translational control of these two viruses. Knocking out CSDE1 severely reduced HRV and PV IRES activity to one-tenth of the control group; this reduction occurs through specifically decreasing IRES activity without changing cap-dependent translation activity. In addition, adding back expression of CSDE1 in $\mathrm{CSDE}^{-/-}$mouse embryonic stem (ES) cells rescued the HRV and PV IRES activity to approximately 69 and 66\%, respectively [41]. Thus, a number of studies have shown that CSDE1 could promote IRES-dependent translation initiation in both eukaryotes and viruses.

In contrast, CSDE1 can also repress cap-independent translation initiation of mRNA. One mRNA target is CSDE1 itself. The CSDE1 protein autoregulates its own expression by repressing IRES activity. In mouse ES cells, the activity of exogenous CSDE1 IRES was 2-fold higher in $\mathrm{CSDE}^{-/-}$cells than in $\mathrm{CSDE}^{+/-}$and $\mathrm{CSDE}^{+/+}$cells. In addition, transfection of exogenous CSDE1 reduced CSDE1 IRES activity by $60 \%$ [48]. Thus, CSDE1 can both promote and repress cap-independent translation initiation. It should be noted that these opposing functions can exist within the same cellular process. For example, during the cell cycle, CSDE1 can increase PITSLRE IRES activity and simultaneously decrease its own IRES activity. This indicates that the homeostasis of CSDE1 bidirectional reprogramming is important for its function.

\section{Promoting and repressing cap-dependent translation initiation}

Although CSDE1 mainly reprograms cap-independent translation initiation, CSDE1 can also stimulate and repress cap-dependent translation initiation. In capdependent translation processes, eukaryotic translation initiation factor 4G (eIF4G) and poly(A)-binding protein 1 (PABP1) interact with each other to promote the circularization of terminal ribosomes from the 3' end to the 5' end [49-51]. Through enhancing the eIF4GPABP1 interaction, CSDE1 increased the interaction between the 5' cap and poly(A) tail to promote capdependent translation. Depletion of CSDE1 led to less eIF4G being pulled down by PABP1 from both HeLa and U2OS cells [52]. Additionally, a dual luciferase reporter assay showed that the cap-dependent translation initiation activity was reduced by $33 \%$ in CSDE1depleted cells [52]. A recent study proposed another mechanism to explain how CSDE1 promoted capdependent translation initiation. It showed that CSDE1 defined a novel class of nucleoplasmic reticulum (NR) named CSDE1-NR, where CSDE1 foci were concentrated. CSDE1-NRs concentrated poly(A) RNAs, ribosomes and translation factors (such as eIF4E) to facilitate cap-dependent translation initiation. Based on ribopuromycylation method analysis, more than $80 \%$ of CSDE1-NRs were puromycin positive, which could be directly visualized through localized translation in the cancer cell line BeWo [24]. Through regulating translation factors, CSDE1 plays an active role in the capdependent translational reprogramming process.

On the other hand, the cap-dependent translation initiation of some specific mRNAs is frequently repressed by sequence-special RNA-binding proteins, which bind to response elements in the 5'UTR or 3'UTRs of RNA [29]. CSDE1 can repress the translation of PABP and male-specific lethal-2 (msl-2) by this mechanism. PABP protein autoregulated its expression by binding the adenine-rich autoregulatory sequence (ARS) in the 5'UTR of PABP mRNA, thus creating a negative feedback mechanism [53]. The binding of CSDE1 and insulin-like growth factor II mRNA binding protein-1 (IMP1) to the ARS element maximally repressed translation of PABP mRNA in a cell-free RRL system [54]. CSDE1 and IMP1 stalled the movement of the 40S preinitiation complex on the PABP 5'UTR [51, 54]. Msl-2 was found to play a key role in dosage compensation of drosophila, which occurs by increasing transcription of the single male $\mathrm{X}$ chromosome by 2 -fold in males and repressing it in females. This process requires the binding of the dosage compensation complex (DCC) to hundreds of sites along the male $\mathrm{X}$ chromosome, and msl-2 is the key component of the DCC $[55,56]$. In females, CSDE1 was recruited by sex lethal (SXL) to msl-2 mRNA to repress its cap-dependent translation initiation [13]. Depleting CSDE1 restored the repressed translation, while the addition of recombinant CSDE1 could rerepress translation in a dose-dependent manner [14].

\section{Increasing and decreasing RNA abundance}

RNA abundance directly influences protein production, so RNA abundance is a crucial component of translation control. The stability of RNA is one of the key factors determining RNA abundance. CSDE1 plays a bidirectional 
translational reprogramming role for RNAs by both increasing and decreasing mRNA stability.

Existing studies indicate that CSDE1 could increase the abundance of some RNAs by maintaining their stability. CSDE1 tends to increase the RNA stability of some targeted RNAs including PTH, c-fos and lncBC200 via binding to the special element of theses RNAs. During the posttranscriptional regulation of parathyroid hormone (PTH) expression, some factors formed an RNA-binding complex on a defined cis-acting instability element in the 3'UTR to protect PTH mRNA from degradation [57]. As one key functional component of the stabilizing complex, CSDE1 contributes to the regulation of PTH mRNA abundance through attaching to the cis-acting instability element in the 3'UTR [58]. In HEK293 cells, overexpression of CSDE1 increased the full-length PTH mRNA abundance by more than 2-fold, while depletion of CSDE1 resulted in a decrease of PTH abundance [58]. Another mechanism comes from c-fos, which is a marker of neuronal activation [59]. Stability of c-fos mRNA is controlled by a special element major protein-coding-region determinant (mCRD). The mCRD-mediated mRNA turnover, can result in rapidly degrading aberrant mRNA [60]. CSDE1 plays a critical role in c-fos stability by binding to the mCRD. In NIH3T3 cells, knocking down CSDE1 decreased the rate of c-fos mRNA decay mediated by mCRD [61]. Overexpression of CSDE1 in NIH3T3 cells had a stabilizing effect on c-fos mRNA [61, 62]. In addition to mRNA, CSDE1 can increase long non-coding RNA (lncRNA) stability by binding to special elements as well. LncBC200 is a 200-nucleotide ncRNA that is normally highly expressed in the brain, but it is aberrantly expressed in various cancers [63]. In MCF-7 cells, CSDE1 could maintain the stability of $1 \mathrm{ncBC} 200$ by binding to the 3' A-rich region, while knocking down CSDE1 reduced the half-life of $\operatorname{lncBC} 200$ by $40 \%$ and decreased its expression [64].

Regarding decreasing mRNA abundance, CSDE1 destabilizes a lot of mRNAs related with differentiation. GATA binding protein 6 (GATA6) is one of the earliest markers of the primitive endoderm (PrE) in the early mouse embryo, and it has a critical role in regulating PrE development [25]. Mouse GATA6 mRNA contains a long 3'UTR with two conserved purine-rich motifs at nucleotides 166-176 and 828-838 (downstream from the stop codon), and they are potential CSDE1-binding motifs. Deletion of CSDE1 in mouse ES cells increased GATA6 mRNA stability by approximately $25 \%$ [12]. By destabilizing GATA6 mRNA, CSDE1 repressed the expression of GATA6 and thus limited embryo stem cell differentiation in a dose-dependent manner. CSDE1 knockout ESCs spontaneously differentiated into PrE, and restoration of CSDE1 expression partially rescued the differentiation ability [12]. Apart from GATA6, CSDE1 also repressed the expression of many other
mRNAs during the process of neural differentiation. Knocking down CSDE1 in human embryonic stem cells (hESCs) resulted in a significant increase of the level of many mRNA targets including FABP7, VIM and so on [65]. In addition, a lot of other CSDE1-bound targets were regulated by CSDE1 in the level of mRNA $[15,26]$.

\section{"Protein-RNA connector" mode}

The role of CSDE1 in translation regulation has been widely and thoroughly investigated $[27,28]$. As discussed above, even within the same biological process, CSDE1 reprograms the translation of different mRNAs in completely opposite directions. The detailed underlying mechanism behind this dual function remains unclear. Based on previous investigations, we found that CSDE1 reprograms translation by acting as a bridge between RNAs and proteins. In most situations, CSDE1 regulates mRNA translation together with other protein partners instead of working alone. Furthermore, CSDE1 does not have regular RNA or protein binding partners. It binds a wide range of RNAs and connects to different protein regulators for each of them. Thus, CSDE1 changes the fate of RNAs by acting as a connector between RNAs and paired protein regulators. This "protein-RNA connector" model can be used to explain its bidirectional role in translational reprogramming (Fig. 1). Based on the specific regulator and the CSDE1 connecting mechanism, this model can be further separated into three forms: sequential connections, mutual connections and facilitating connections.

\section{Sequential connections}

In the sequential connection mechanism, translational regulators cannot bind directly with RNA. They interact with targeted RNAs via binding to CSDE1. The protein regulator, CSDE1 and the RNA form a sequential connection model of "regulator-CSDE1-RNA". CSDE1 acts as a bridge to connect RNAs and proteins that cannot bind directly to each other.

The function of serine/threonine kinase receptor associated protein (Strap) on RNAs can be explained by this mechanism. Strap is also named Unrip due to its interaction with CSDE1 [66]. Unrip had no inherent RNA binding capacity, but CSDE1 bridges Unrip with target RNAs [67]. One example is $\operatorname{lncBC} 200$, a long noncoding RNA that directly and indirectly interacts with CSDE1 and Unrip, respectively. CSDE1 binds to the $3^{\prime}$ A-rich region of $\operatorname{lncBC} 200$. Unrip indirectly binds to $\operatorname{lncBC} 200$ via heterodimerization with CSDE1. Immunoprecipitation assays showed that knocking down Unrip had a negligible impact on lncBC200 binding to CSDE1. However, knocking down CSDE1 in MCF-7 cells reduced Unrip binding on lncBC200 by more than $80 \%$ [64]. These results suggest the existence of a sequential connection of "Unrip-CSDE1-lncBC200". 


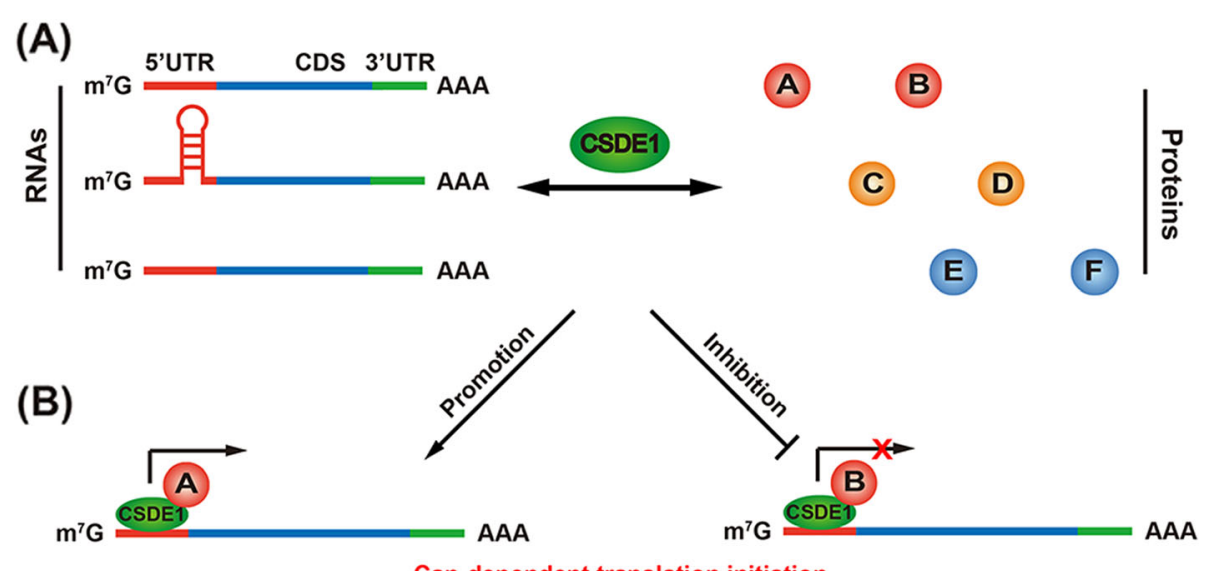

Cap-dependent translation initiation
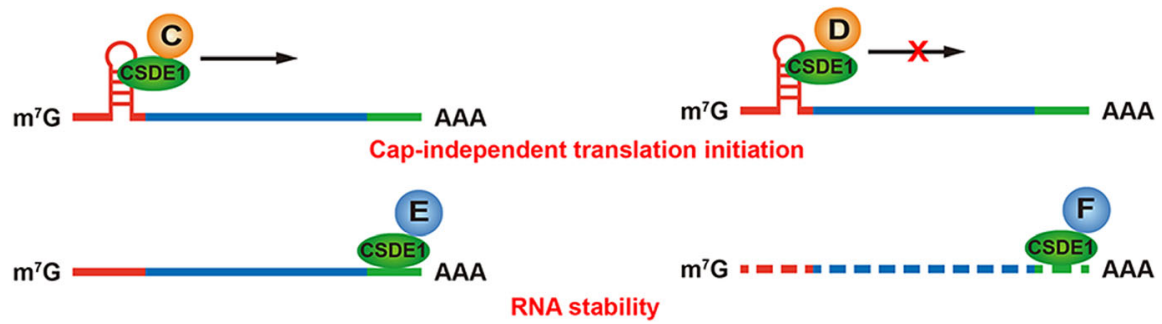

Fig. 1 The bidirectional role of CSDE1 in translational reprogramming. (A) CSDE1 connects different protein regulators (A-F) with target RNAs to change the fate of RNAs. (B) The bidirectional role of CSDE1 in translational reprogramming. CSDE1 not only promotes and represses capdependent and cap-independent translation initiation of RNAs but also increases and decreases RNA abundance

Another example is HRV-2. Unrip and CSDE1 form a sequential connection of "Unrip-CSDE1-HRV2" to stimulate HRV-2 IRES activity and promote its translation initiation in MLE cells. Additional studies reported that Unrip regulated the translation of more CSDE1-bound transcripts, including hydroxymethylbilane synthase (Hmbs), eukaryotic translation initiation factor 4G3 (eIF4G3), poly(A) binding protein cytoplasmic 4 (PABPC4) and so on [68].

The sequential connection mode is applicable for translational regulators binding mRNA indirectly through CSDE1. In this mode, CSDE1 provides proteins with a platform for translational reprogramming. However, the mechanism is less reported.

\section{Mutual connections}

For the translation regulators with the ability to bind RNA, CSDE1 can enhance the interaction between that protein and its target RNA via binding to both of them. The three then form a trimeric complex with internal mutual interaction to reprogram the translation or stability of bound RNAs. This mechanism has been widely studied.

One of the strongest pieces of evidence for this mechanism is that the CSDE1/SXL complex corepresses the translation of msl-2 in female drosophila. Two repressors, CSDE1 and SXL, can interact with each other, and both can bind to msl-2 mRNA. When repressing msl-2 translation, the female-specific RNA binding protein SXL binds to the
3'UTR of msl-2 mRNA and recruits CSDE1 to adjacent regulatory sequences $[14,69-71]$. Then, CSDE1 formed a co-repressor complex with SXL to inhibit ribosome recruitment, which represses msl-2 mRNA translation [14, 70, 72]. The purine-rich sequence downstream of AGCACGUG (nucleotides 9-16) was sandwiched by the CSD1 domain of CSDE1 and the RBD3 domain of SXL [70]. The mutual connection of "CSDE1/SXL/msl-2" played an irreplaceable role in dosage compensation by reprogramming msl-2 translation. Another example is that CSDE1 is involved in the autoregulation of PABP mRNA [51]. CSDE1 can bind both PABP protein and the PABP ARS element directly. However, CSDE1 alone has a lower binding capacity for the ARS than it does when it is acting together with either PABP or IMP1. The presence of PABP can stimulate CSDE1 binding on ARS in a dose-dependent manner. Thus, CSDE1 binds to PABP mRNA and forms a complex with PABP protein during this autoregulation process, and this complex formation results in the repression of PABP expression at the translation level in HeLa cells [51]. Considering the significance of PABP in translation initiation, this mutual connection of "CSDE1/PABP/PABP mRNA" has a crucial role in translation initiation, especially in a capdependent manner. The role of CSDE1 in mCRD-mediated mRNA turnover in NIH3T3 and HeLa cells also supports this mechanism [62]. Deadenylation (shortening of the poly(A) tail) of mRNA is the rate-limiting step of mRNA 
decay and a necessary first step coupled to translation. The $\mathrm{mCRD}$, which is a sequence in the protein-coding region of c-fos mRNA, directs rapid decay of mRNA via deadenylation. CSDE1 binds to the $\mathrm{mCRD}$ and forms a complex with four proteins: PABP, PABP-interacting protein 1 (PAIP-1), heterogeneous nuclear ribonucleoprotein $\mathrm{R}(\mathrm{hnRNP} \mathrm{R})$ and NS1-associated protein 1 (NSAP1) [62]. These proteins prevent deadenylation and RNA decay prior to translation. During the formation of this complex, CSDE1 and PABP bind to the $\mathrm{mCRD}$ and the poly(A) tail of c-fos mRNA, respectively. Thus, the CSDE1-PABP interaction built a "bridge" between the c-fos mCRD and its poly(A) tail. This "bridge" is necessary for "CSDE1/protein complex/c-fos" complex formation, which maintains c-fos mRNA stability. This connection reveals a new mechanism for controlling cell growth and differentiation.

Mechanisms similar to this mutual connection have been reported for some other RBPs in previous investigations. The Ccr4-Not complex controls mRNA decay and translation efficiency by removing mRNA poly(A) tails [73]. The complex consists of a number of proteins, mainly Ccr4, Caf1, Caf40, Caf130, Not1, Not2, Not3, Not4, Not5, Not10 and Not11. Shortening of the 3' poly(A) tail (deadenylation) causes cleavage of the $5^{\prime} \mathrm{m} 7 \mathrm{GpppN}$ cap (decapping) and results in translational repression [74]. The Ccr4-Not complex can be recruited by RBPs to the 3'UTR of target mRNAs to promote mRNA decay. For example, the RBP Mpt5p, which is a member of the PUF protein family, controls HO mRNA stability by this mechanism. Mpt5p recruits the Ccr4-Not complex to the HO mRNA 3'UTR to stimulate deadenylation of HO mRNA by binding to Caf1 [75]. The "Mpt5p/ Ccr4-Not complex/HO mRNA" consists of mutual connections. Another example is that the RBPs Roquin1 and Roquin 2 promote target mRNA degradation by recruiting the Ccr4-Not complex [76]. In addition, the mechanism of some RBPs stimulating translation by recruiting PABP to the RNA poly(A) tail is similar to this mutual connection. As discussed above, PABP binds to a poly(A) tail that has eIFs at the 5 ' end. PABP then brings the mRNA ends into close proximity to effectively circularize the mRNA and promote translation. For example, the DAZL family of proteins, which are germ-cell-specific RNA-binding proteins that are essential for gametogenesis, stimulate translation of target mRNAs by recruiting PABPs to the poly(A) tail [77]. Similarly, the "DAZL/PABP/target mRNAs" form a mutual connection. In summary, for the mutual connection model, CSDE1 enhances RNA-protein interactions by interacting with them both. This model is the most important and widely existing mechanism in CSDE1-mediated translation initiation reprogramming.

\section{Facilitating connections}

Some translation regulators can bind to RNA. However, some translational regulators need the help of CSDE1, which acts as a pioneer, paving the way for their binding. With this mechanism, CSDE1 facilitates the binding of regulators and RNAs rather than interacting with regulators directly. CSDE1 changes the structure of bound RNA and makes it more accessible for other protein regulators to bind.

CSDE1 mediates both cellular and virus IRES activity based on this mechanism. Among these different mechanisms, the role of CSDE1 in regulating Apaf- 1 and HRV-2 translation initiation is the most widely studied [39, 42]. Both Apaf-1 and HRV-2 mRNA contain IRES elements. Two proteins, CSDE1 and PTB, bind to the IRES elements separately to make the ribosome loading site in the IRES more accessible. Then, CSDE1 and PTB promote IRESmediated ribosome recruitment and facilitate translation initiation of the bound mRNAs [39, 42]. For the cellular IRES of Apaf-1, CSDE1 first binds to a purine-rich region within a stem-loop structure [39]. This CSDE1 binding forms a structure that permits PTB binding on another exposed loop in the Apaf-1 IRES. Thus, CSDE1 and PTB both bind to the Apaf-1 IRES and promote IRESdependent translation initiation [39]. For HRV-2, PTB neither interacts with CSDE1 nor affects the binding of CSDE1 to HRV-2 [42]. However, the interaction of PTB with HRV-2 is affected by CSDE1 and HRV-2 binding. In vitro assays showed that CSDE1 bound to subdomains 2 and 5 of the HRV-2 IRES and brought them close together to change the IRES structure [42]. This paved the way for PTB to easily bind. Generally, CSDE1 facilitates PTB binding of Apaf-1 and HRV-2 mRNA, meaning that CSDE1 promotes their translation initiation together with PTB. This facilitating connection mode also exists in noncoding RNAs. The protein Maleless (MLE) and two noncoding RNAs (roX1 and roX2) are components of the dosage compensation complex (DCC) [78]. In dosage compensation, CSDE1 facilitated the binding of MLE to the two roX RNAs and subsequently enhances MLE-roX interaction, which promoted DCC assembly on the chromosome in male Drosophila [79]. This facilitated connection mode is an interesting mechanism. Although CSDE1 cannot bind with these protein regulators, CSDE1 is essential for the regulator binding to the RNAs [42]. Although CSDE1 and the protein regulators bind at two different positions of the RNA with long distances between them, they can still work together to reprogram mRNA translation [39, 42].

In summary, CSDE1 acts as a "protein-RNA connector" in bidirectional reprogramming by three different mechanisms (Fig. 2). As indicated above, the mutual connection mode is the most common. In this mechanism, CSDE1, protein regulators and RNAs bind tightly with each other and form a protein-RNA complex to regulate RNA translation and stability [14, 62]. The facilitating connection mode is the second most common mechanism. In this mechanism, CSDE1 facilitates the 


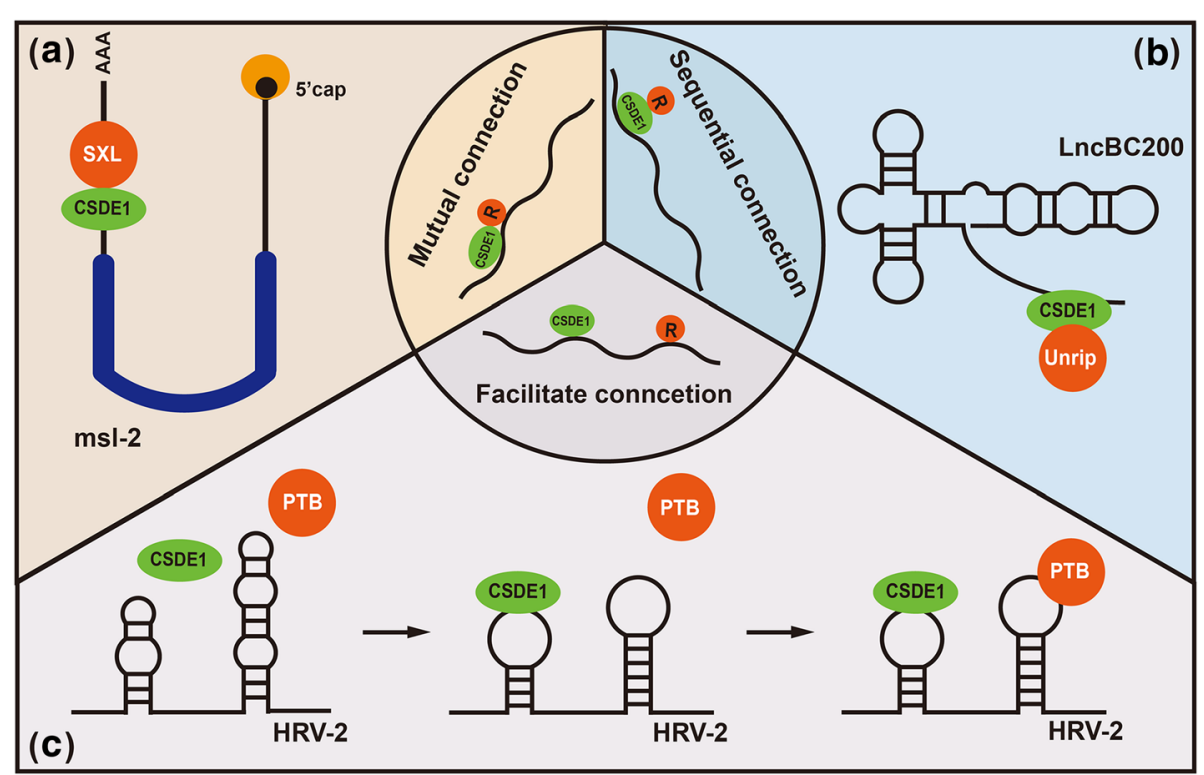

Fig. 2 The "protein-RNA connector" model. The "protein-RNA connector" model can be used to explain the bidirectional role of CSDE1 in translational reprogramming. Based on the interactions among CSDE1, regulators and RNAs, this model can be further divided into three forms: sequential connection, mutual connection and facilitating connection. a For sequential connection, CSDE1 mediates a protein regulator binding indirectly to RNA. As an example, Unrip binds indirectly to IncBC200 via interacting with CSDE1, which enhances the abundance of IncBC200. b For mutual connection, a complex forms among RNA, CSDE1 and protein regulator. As an example, CSDE1, SXL and msl-2 all bind to one another to repress the translation of msl-2. c To facilitate connections, CSDE1 binds to RNA to enable the binding of other regulators. As an example, CSDE1 binds to HRV-2 mRNA and changes its structure to facilitate PTB binding, which promotes the translation of HRV-2

binding of other regulators to RNA by adjusting the IRES structure [39, 42]. In a few cases, CSDE1 connects indirect regulators with their target RNAs based on the sequential connection mode [64]. For all modes, CSDE1 binds RNAs directly and cooperates with other regulators in direct or indirect ways. CSDE1 connects different RNAs with their regulators to achieve different purposes (Table 2). The complex of CSDE1, regulator and RNA enable reprogramming of the translation initiation in different directions for each transcript $[11,14]$. Thus, CSDE1 plays a bidirectional regulatory role in different biological processes.

\section{Role of CSDE1 in human disease}

As a critical factor in translational reprogramming, CSDE1 controls the translation of a number of mRNAs that are correlated with various diseases. Previous studies suggest that a pathological status can be reprogrammed by CSDE1 via changing translation of a subset of mRNAs. CSDE1 is emerging as a potential drug target and prognostic biomarker for some diseases (Table 1).

\section{Cancers}

Cancers are the most widely and thoroughly investigated diseases related to CSDE1. CSDE1 has been shown to be a potential drug target, prognostic biomarker and anticancer drug sensitizer for various cancers.

CSDE1 is an oncogene in melanoma, and suppressing CSDE1 can reduce tumour malignancy in both cancer cells and animal models. CSDE1 expression is higher in a variety of melanoma cell lines, including SK-Mel-19, SK-

Table 2 The components and function of CSDE1 in the "Protein-RNA connector" model

\begin{tabular}{|c|c|c|c|c|c|c|}
\hline Connection models & Protein regulators & RNA & CSDE1 function & Reprogramming directions & Cells & References \\
\hline Sequential & Unrip & BC200 & RNA stability & $\uparrow$ & MCF-7 & [64] \\
\hline \multirow[t]{3}{*}{ Mutual } & $S X L$ & Msl-2 & Translation & $\downarrow$ & Drosophila female & {$[14,69,70]$} \\
\hline & IMP1,PABP & PABP & Translation & $\downarrow$ & HeLa & {$[51,54]$} \\
\hline & PABP, PAIP-1, hnRNP D, NSAP1 & $c-f o s$ & RNA stability & $\uparrow$ & NIH3T3; HeLa & [62] \\
\hline \multirow[t]{3}{*}{ Facilitate } & PTB & Apaf-1 & Translation & $\uparrow$ & COS7, MRC5, SY5Y, BALB/C & [11] \\
\hline & PTB & HRV-2 & Translation & $\uparrow$ & HeLa & [42] \\
\hline & PTB & CSDE1 & Translation & $\downarrow$ & Mouse ES & [48] \\
\hline
\end{tabular}


Mel-29, and SK-Mel-103, than in normal melanocytes [15]. In patients, CSDE1 was also higher in both primary and metastatic malignant melanoma tumour samples than it was in benign nevi, as shown by immunohistochemistry (IHC). Additionally, depletion of CSDE1 decreased melanoma tumour growth and metastatic capacity, and overexpression of CSDE1 promoted migration and invasion. Studies in a mouse model further suggested that depletion of CSDE1 strikingly reduced the metastasis to the lung, which is a frequent metastatic site for melanoma [15]. In human colorectal cancer (CRC), CSDE1 promoted cancer cell survival, invasion, and resistance to apoptosis [80]. CSDE1 was also overexpressed by an average of almost 3fold in tumour samples compared with their adjacent normal tissues in CRC patients. Overexpressed CSDE1 was correlated with poor prognosis in patients. Furthermore, knocking down CSDE1 in CRC cells decreased viability and the migration ratio by approximately $40 \%$. As a treatment, downregulating CSDE1 increased the camptothecin response in CRC-derived cell lines and CRC patients [80].

High expression of CSDE1 was associated with poor prognosis in glioma and pancreatic ductal adenocarcinoma (PDAC) [16]. CSDE1 expression was also significantly higher in glioma cells and tissues than it was in normal human astrocytes and brain tissues. The CSDE1 mRNA expression level was also higher in grade IV glioma than it was in grade II and III patients $(P<0.001)$ according to the data released by the Chinese Glioma Genome Atlas (CGGA). In addition, higher CSDE1 expression was correlated with poorer survival in glioma patients $(P=0.0177)[16]$. Another recent investigation revealed that low CSDE1 expression was significantly associated with poor outcome in low-grade resected PDAC patients [20]. Based on this study, CSDE1 was used as an independent prognostic biomarker for resectable pancreatic cancer. In addition, for breast cancer, which is the most common cancer affecting women [81], CSDE1 mRNA expression was nearly 10-fold higher in MCF-7 breast cancer cells than in normal cell lines [17].

However, CSDE1 plays an opposing role in some other types of tumours. Pheochromocytomas (PCCs) and paragangliomas (PGLs) are neuroendocrine tumours with low incidence but high mortality [82, 83]. CSDE1 may play a tumour suppressor role in PCCs/PGLs. Loss of function mutations in CSDE1 emerged as a driver of PCC/PGL tumourigenesis. Multi-platform integration, including whole-exome sequencing and mRNA sequencing, revealed that a somatic CSDE1 loss of function mutation drove progression of PCCs/PGLs. CSDE1mutated tumours exhibited a CSDE1 DNA copy number deletion and inadequate CSDE1 mRNA expression, which supported its role as a tumour suppressor in tumourigenesis [18]. Oral squamous cell carcinoma (OSCC) is one of the most widely occurring cancers and is affected by genetic alternations [84, 85]. A research screen for oxidative stress-related genes revealed that the expression level of CSDE1 was downregulated (median fold change $=0.769)$ in OSCC $(P=0.043)$ [19].

In addition, CSDE1 was identified as a possible platinum drug sensitizer. Platinum-based chemotherapy is a first-line treatment for epithelial ovarian cancer (EOC). However, approximately $25 \%$ of patients are resistant to platinum therapies within 6 months [86]. It was previously reported that some key translation regulation factors played an important role in affecting platinum sensitization $[87,88]$, and CSDE1 was found to be involved in regulating platinum sensitization of tumours. Silencing CSDE1 caused significant sensitization to platinum in four epithelial ovarian cancer cell lines, SKOV-3, CAOV-3, ES-2 and OVCAR-3. When CSDE1 was silenced, the sensitivity increased by 11 to $50 \%$ over what was observed in control cells, which indicated that CSDE1 might be an anticancer drug sensitizer [21]. Furthermore, CSDE1 inhibition increases EOC cell sensitivity to platinum.

\section{Other diseases}

With recent research advances, CSDE1 has been found to play an important role in other diseases, including Diamond-Blackfan anaemia (DBA), autism spectrum disorders (ASDs) and embryonic lethality.

DBA is a rare bone marrow failure disease with a paucity of erythroid precursors [89]. More than half of DBA patients carry mutations in genes encoding ribosomal protein S19 (Rps19) or L11 (Rpl11). A polysome profiling assay showed that the translation of CSDE1 was repressed when Rps19 was lost. In erythroblasts from DBA patients, CSDE1 expression was 3-fold lower than it was in control cells. Decreased CSDE1 expression inhibited erythroid proliferation and differentiation. CSDE1depleted cells mainly became pyknotic and failed to mature to enucleated erythrocytes [23]. Thus, increasing CSDE1 expression may reverse impaired erythroid proliferation and differentiation.

ASDs are harmful and have a high incidence, with a prevalence of approximately 1-2\% [90]. ASD occurrence is largely attributed to genetic variation, especially copy number variations (CNVs) [91]. However, currently known CNVs are mostly rare and account for only a small proportion of cases. Whole exome sequencing of 918 individuals found that a de novo loss of function mutation in CSDE1 was strongly associated with autism [92]. In addition, another genome-wide association study on autism that used two Chinese cohorts for discovery $(n=2150)$ and three data sets of European ancestry populations for validation suggested that CSDE1 was a candidate gene for autism $\left(P=5.51 \times 10^{-6}\right)$ [22]. These results show that CSDE1 may be one of the causal genes of ASDs, although the detailed mechanism still needs further investigation. 
Embryonic development is one of the most important stages of life. In both knockout mice and knockout embryonic stem cells (ECS), CSDE1 was identified as critical for placental development [12, 24]. Lacking CSDE1 resulted in embryonic lethality at mid-gestation. In CSDE1-KO mouse placentas, the spongiotrophoblast and labyrinthine layers showed marked atrophy, and the number of trophoblast giant cells decreased by approximately $60-75 \%$ [24]. More importantly, the expression of CSDE1 was significantly higher inhESCs than in their differentiated neural progenitor cells (NPCs) and neuronal counterparts. In addition, CSDE1 protein levels decrease during the hESC differentiation process. CSDE1 expression impairs the neural differentiation of hESCs. Knocking down CSDE1 in hESCs facilitated neural differentiation, and overexpression of CSDE1 impaired neural differentiation by regulating the stability and translation of fatty acid binding protein 7 (FABP7) mRNA as well as the stability of vimentin (VIM) [65]. Therefore, CSDE1 is one of the decisive factors for embryonic development and plays an important role in embryonic reprogramming.

\section{Conclusions}

CSDE1 plays a special bidirectional role in translational reprogramming by regulating the translation initiation and abundance of various RNAs. Among its targets, some RNAs are cell type specific, such as Apaf- 1 .

Although translational reprogramming is a very complex event, some key factors determine the change of translation during these biological processes [93-95]. We propose that these factors can be considered translational reprogramming points. They determine the expression levels of different mRNAs by changing their translational fate. In our view, CSDE1 acts at special bidirectional translational reprogramming points in various biological processes. The occurrence and development of many diseases are based on the impaired homeostasis of key proteins. As discussed above, CSDE1 determines the expression of many diseaserelated mRNAs. The bidirectional role of CSDE1 in translation reprogramming contributes to its bidirectional role in cancers: CSDE1 can act as both an oncogene and a tumour suppressor in different tumours. In addition, CSDE1 is emerging as a potential biomarker and therapeutic target in various human diseases. Thus, we propose the "proteinRNA connector" model to explain its bidirectional role. In this molecular model, CSDE1 increases the interaction between RNAs and the regulators, which changes the fate of the RNA, the cell and even the biological processes. This "protein-RNA connector" model helps us to understand the role of CSDE1 in disease reprogramming.

However, there are more areas awaiting investigation. First, the translation reprogramming role of CSDE1 is still not explored at the genome-wide scale. As a connector,
CSDE1 should bind to a number of RNAs and proteins to regulate expression at the transcriptome and proteome level. Currently, it is unclear which RNAs are reprogrammed by CSDE1 at the translational level and which protein regulators are connected to specific RNAs by CSDE1 during the translational reprogramming process. Second, the role of CSDE1 in translation homeostasis largely remains unknown. Although CSDE1 bidirectionally reprograms translation within single biological and pathological processes, these processes should maintain homeostasis. Under unbalanced conditions, CSDE1 should be capable of restoring homeostasis by regulating the direction of reprogramming. This role of CSDE1 in stress response needs further investigation. Finally, the role of CSDE1 in human diseases is emerging. It is still unknown how many diseases are correlated with CSDE1-mediated translation reprogramming. The clinical roles for CSDE1 in disease reprogramming, as a predictive biomarker, and as a drug target and sensitizer still need to be confirmed.

\section{Abbreviations}

Apaf-1: Apoptotic peptidase activating factor 1; ARS: Autoregulatory sequence; ASD: Autism spectrum disorder; CGGA: Chinese Glioma Genome Atlas; CNVs: Copy number variations; CRC: Colorectal cancer; CSDE1: Cold shock domain containing E1; DBA: Diamond-Blackfan anemia; DCC: Dosage compensation complex; ECS: Embryonic stem cells; elF4G: Eukaryotic translation initiation factor 4G; EOC: Epithelial ovarian cancer; FABP7: Fatty acid binding protein 7; GATA6: GATA binding protein 6; hESCs: Human embryonic stem cells; Hmbs: Hydroxymethylbilane synthase; hnRNP R: Heterogeneous nuclear ribonucleoprotein R; hnRNPK: Heterogeneous nuclear ribonucleoprotein K; HRV: Human rhinovirus;

IHC: Immunohistochemistry; IMP1: Insulin-like growth factor II mRNA binding protein-1; IRES: Internal ribosome entry site; ITAFs: IRES trans-acting factors; IncRNA: Long non-coding RNA; mCRD: Major protein-coding-region determinant; MLE: Maleless; msl-2: Male specific lethal-2; NPC: Neural progenitor cell; NR: Nucleoplasmic reticulum; NSAP1: NS1-associated protein 1; OSCC: Oral squamous cell carcinoma; PABP1: Poly(A)-binding protein 1; PABPC4: Poly(A) binding protein cytoplasmic 4; PAIP-1: PABP-interacting protein 1; PCBP1: Poly $(\mathrm{rC})$ binding protein 1; PCBP2: Poly $(\mathrm{rC})$ binding protein 2; PCCs: Pheochromocytomas; PDAC: Pancreatic ductal adenocarcinoma; PGLs: Paragangliomas; PrE: Primitive endoderm; PTH: Parathyroid hormone; PV: Poliovirus; RBP: RNA-binding protein; RP: Ribosomal protein; RRL: Rabbit reticulocyte lysate; Strap: Serine/threonine kinase receptor associated protein; SXL: Sex lethal; UNR: Upstream of N-RAS; Unrip: UNR-interacting protein; UTR: Untranslated region; VIM: Vimentin

\section{Acknowledgements}

We thank for Ke Liu and Fan Xiao for their discussion of the models.

\section{Authors' contributions}

A-XG drafted the manuscript, J-JC and L-YW assisted in the writing, J-YY edited the manuscript to its final version. All authors read and approved the final manuscript.

\section{Authors' information}

$\mathrm{Ji-Ye} \mathrm{Yin} \mathrm{is} \mathrm{a} \mathrm{professor} \mathrm{of} \mathrm{pharmacology} \mathrm{in} \mathrm{Department} \mathrm{of} \mathrm{Clinical}$ Pharmacology, Xiangya Hospital, Central South University. Ao-Xiang Guo, JiaJia Cui and Lei-Yun Wang are students in Department of Clinical Pharmacology, Xiangya Hospital, Central South University.

Funding

This work was supported by the National Natural Science Foundation of China $(81773823,81573463)$ and National Major Science and Technology Project (2017ZX09304014, 2016YFC0905000). 


\section{Availability of data and materials}

Not applicable.

\section{Ethics approval and consent to participate \\ Not applicable.}

\section{Consent for publication}

Not applicable.

\section{Competing interests}

The authors declare that they have no competing interests.

\section{Author details}

'Department of Clinical Pharmacology, Xiangya Hospital, Central South University, Changsha 410078, People's Republic of China. ${ }^{2}$ Institute of Clinical Pharmacology, Central South University; Hunan Key Laboratory of Pharmacogenetics, Changsha 410078, People's Republic of China. ${ }^{3}$ Engineering Research Center of Applied Technology of Pharmacogenomics, Ministry of Education, 110 Xiangya Road, Changsha 410078, People's Republic of China. ${ }^{4}$ National Clinical Research Center for Geriatric Disorders, 87 Xiangya Road, Changsha 410008, Hunan, People's Republic of China. ${ }^{5}$ Hunan Provincial Gynecological Cancer Diagnosis and Treatment Engineering Research Center, Changsha 410078, People's Republic of China ${ }^{6}$ Hunan Key Laboratory of Precise Diagnosis and Treatment of Gastrointestinal Tumor, Changsha 410078, People's Republic of China.

Received: 3 September 2019 Accepted: 16 December 2019 Published online: 27 January 2020

\section{References}

1. Signor SA, Nuzhdin SV. The evolution of gene expression in cis and trans. Trends Genet. 2018;34(7):532-44.

2. Noormohammadi A, Calculli G, Gutierrez-Garcia R, Khodakarami A, Koyuncu S, Vilchez D. Mechanisms of protein homeostasis (proteostasis) maintain stem cell identity in mammalian pluripotent stem cells. Cell Mol life Sci. 2018;75(2):275-90.

3. Su X, Yu Y, Zhong Y, Giannopoulou EG, Hu X, Liu H, et al. Interferon-gamma regulates cellular metabolism and mRNA translation to potentiate macrophage activation. Nat Immunol. 2015;16(8):838-49.

4. Andreev DE, O'Connor PB, Zhdanov AV, Dmitriev RI, Shatsky IN, Papkovsky $D B$, et al. Oxygen and glucose deprivation induces widespread alterations in mRNA translation within 20 minutes. Genome Biol. 2015;16:90.

5. Starck SR, Tsai JC, Chen K, Shodiya M, Wang L, Yahiro K, et al. Translation from the $5^{\prime}$ untranslated region shapes the integrated stress response. Science (New York, NY). 2016;351(6272):aad3867.

6. Jacquemin-Sablon $\mathrm{H}$, Dautry F. Organization of the unr/N-ras locus: characterization of the promoter region of the human unr gene. Nucleic Acids Res. 1992:20(23):6355-61.

7. Graumann PL, Marahiel MA. A superfamily of proteins that contain the coldshock domain. Trends Biochem Sci. 1998;23(8):286-90.

8. Dormoy-Raclet V, Markovits J, Malato Y, Huet S, Lagarde P, Montaudon D, et al. Unr, a cytoplasmic RNA-binding protein with cold-shock domains, is involved in control of apoptosis in ES and HuH7 cells. Oncogene. 2007; 26(18):2595-605

9. Yin JY, Dong ZZ, Liu RY, Chen J, Liu ZQ, Zhang JT. Translational regulation of RPA2 via internal ribosomal entry site and by elF3a. Carcinogenesis. 2013; 34(6):1224-31.

10. Schepens B, Tinton SA, Bruynooghe Y, Parthoens E, Haegman M, Beyaert R, et al. A role for hnRNP C1/C2 and Unr in internal initiation of translation during mitosis. EMBO J. 2007;26(1):158-69.

11. Mitchell SA, Brown EC, Coldwell MJ, Jackson RJ, Willis AE. Protein factor requirements of the Apaf-1 internal ribosome entry segment: roles of polypyrimidine tract binding protein and upstream of N-ras. Mol Cell Biol. 2001;21(10):3364-74

12. Elatmani H, Dormoy-Raclet V, Dubus P, Dautry F, Chazaud C, JacqueminSablon $\mathrm{H}$. The RNA-binding protein Unr prevents mouse embryonic stem cells differentiation toward the primitive endoderm lineage. Stem cells (Dayton, Ohio). 2011;29(10):1504-16.

13. Patalano S, Mihailovich M, Belacortu Y, Paricio N, Gebauer F. Dual sex-specific functions of Drosophila Upstream of $\mathrm{N}$-ras in the control of $\mathrm{X}$ chromosome dosage compensation. Dev (Cambridge, England). 2009;136(4):689-98.
14. Abaza I, Coll O, Patalano S, Gebauer F. Drosophila UNR is required for translational repression of male-specific lethal 2 mRNA during regulation of X-chromosome dosage compensation. Genes Dev. 2006;20(3):380-9.

15. Wurth L, Papasaikas P, Olmeda D, Bley N, Calvo GT, Guerrero S, et al. UNR/ CSDE1 drives a post-transcriptional program to promote melanoma invasion and metastasis. Cancer Cell. 2016;30(5):694-707.

16. Tian NY, Qi YJ, Hu Y, Yin B, Yuan JG, Qiang BQ, et al. RNA-binding Protein UNR Promotes Glioma Cell Migration and Regulates the Expression of Ribosomal Protein L9. Chin Med Sci J. 2018;33(3):143-51.

17. Fang $H$, Yue $X$, Li X, Taylor JS. Identification and characterization of high affinity antisense PNAs for the human unr (upstream of N-ras) mRNA which is uniquely overexpressed in MCF-7 breast cancer cells. Nucleic Acids Res. 2005:33(21):6700-11.

18. Fishbein $L$, Leshchiner I, Walter $V$, Danilova $L$, Robertson AG, Johnson AR, et al. Comprehensive molecular characterization of Pheochromocytoma and Paraganglioma. Cancer Cell. 2017;31(2):181-93.

19. Pedro NF, Biselli JM, Maniglia JV, Santi-Neto D, Pavarino EC, Goloni-Bertollo EM, et al. Candidate biomarkers for Oral squamous cell carcinoma: differential expression of oxidative stress-related genes. Asian Pac J Cancer Prev. 2018;19(5):1343-9.

20. Martinez-Useros J, Georgiev-Hristov T, Fernandez-Acenero MJ, BorreroPalacios A, Indacochea A, Guerrero S, et al. UNR/CDSE1 expression as prognosis biomarker in resectable pancreatic ductal adenocarcinoma patients: a proof-of-concept. PLoS One. 2017;12(8):e0182044.

21. Penzvalto Z, Lanczky A, Lenart J, Meggyeshazi N, Krenacs T, Szoboszlai N, et al. MEK1 is associated with carboplatin resistance and is a prognostic biomarker in epithelial ovarian cancer. BMC Cancer. 2014;14:837.

22. Xia K, Guo H, Hu Z, Xun G, Zuo L, Peng Y, et al. Common genetic variants on 1p13.2 associate with risk of autism. Mol Psychiatry. 2014;19(11):1212-9.

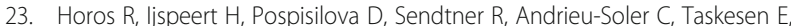
et al. Ribosomal deficiencies in diamond-Blackfan anemia impair translation of transcripts essential for differentiation of murine and human erythroblasts. Blood. 2012;119(1):262-72.

24. Saltel F, Giese A, Azzi L, Elatmani $H$, Costet $P$, Ezzoukhry Z, et al. Unr defines a novel class of nucleoplasmic reticulum involved in mRNA translation. J Cell Sci. 2017;130(10):1796-808.

25. Schrode N, Saiz N, Di Talia S, Hadjantonakis AK. GATA6 levels modulate primitive endoderm cell fate choice and timing in the mouse blastocyst. Dev Cell. 2014;29(4):454-67.

26. Moore KS, Yagci N, van Alphen F, Paolini NA, Horos R, Held NM, et al. Csde1 binds transcripts involved in protein homeostasis and controls their expression in an erythroid cell line. Sci Rep. 2018;8(1):2628.

27. Ray S, Catnaigh PO, Anderson EC. Post-transcriptional regulation of gene expression by Unr. Biochem Soc Trans. 2015;43(3):323-7.

28. Anderson EC, Catnaigh PO. Regulation of the expression and activity of Unr in mammalian cells. Biochem Soc Trans. 2015;43(6):1241-6.

29. Sonenberg N, Hinnebusch AG. Regulation of translation initiation in eukaryotes: mechanisms and biological targets. Cell. 2009;136(4):731-45.

30. Hinnebusch AG. The scanning mechanism of eukaryotic translation initiation. Annu Rev Biochem. 2014;83:779-812.

31. Hinnebusch AG, Ivanov IP, Sonenberg N. Translational control by 5'untranslated regions of eukaryotic mRNAs. Science (New York, NY). 2016; 352(6292):1413-6.

32. Philippe C, Dubrac A, Quelen C, Desquesnes A, Van Den Berghe L, Segura C, et al. PERK mediates the IRES-dependent translational activation of mRNAs encoding angiogenic growth factors after ischemic stress. Sci Signal. 2016;9(426):ra44.

33. Siculella L, Tocci R, Rochira A, Testini M, Gnoni A, Damiano F. Lipid accumulation stimulates the cap-independent translation of SREBP-1a mRNA by promoting hnRNP A1 binding to its $5^{\prime}$-UTR in a cellular model of hepatic steatosis. Biochim Biophys Acta. 2016;1861(5):471-81.

34. Shi Y, Yang Y, Hoang B, Bardeleben C, Holmes B, Gera J, et al. Therapeutic potential of targeting IRES-dependent c-myc translation in multiple myeloma cells during ER stress. Oncogene. 2016;35(8):1015-24.

35. Shatsky IN, Terenin IM, Smirnova W, Andreev DE. Cap-independent translation: What's in a name? Trends Biochem Sci. 2018;43(11):882-95.

36. Yamamoto H, Unbehaun A, Spahn CMT. Ribosomal chamber music: toward an understanding of IRES mechanisms. Trends Biochem Sci. 2017;42(8):655-68.

37. Godet AC, David F, Hantelys F, Tatin F, Lacazette E, Garmy-Susini B, Prats AC. IRES Trans-Acting Factors, Key Actors of the Stress Response. Int. J. Mol. Sci. 2019;20:924.

38. Weingarten-Gabbay S, Elias-Kirma S, Nir R, Gritsenko AA, Stern-Ginossar N, Yakhini Z, et al. Comparative genetics. Systematic discovery of cap- 
independent translation sequences in human and viral genomes. Science (New York, NY). 2016;351(6270):aad4939. https://doi.org/10.1126/science.aad4939.

39. Mitchell SA, Spriggs KA, Coldwell MJ, Jackson RJ, Willis AE. The Apaf-1 internal ribosome entry segment attains the correct structural conformation for function via interactions with PTB and unr. Mol Cell. 2003;11(3):757-71.

40. Tinton SA, Schepens B, Bruynooghe Y, Beyaert R, Cornelis S. Regulation of the cell-cycle-dependent internal ribosome entry site of the PITSLRE protein kinase: roles of Unr (upstream of N-ras) protein and phosphorylated translation initiation factor elF-2alpha. Biochem J. 2005;385(Pt 1):155-63.

41. Boussadia O, Niepmann M, Creancier L, Prats AC, Dautry F, JacqueminSablon $\mathrm{H}$. Unr is required in vivo for efficient initiation of translation from the internal ribosome entry sites of both rhinovirus and poliovirus. J Virol. 2003;77(6):3353-9.

42. Anderson EC, Hunt SL, Jackson RJ. Internal initiation of translation from the human rhinovirus-2 internal ribosome entry site requires the binding of Unr to two distinct sites on the 5' untranslated region. J Gen Virol. 2007:88(Pt 11):3043-52.

43. Coldwell MJ, Mitchell SA, Stoneley M, MacFarlane M, Willis AE. Initiation of Apaf-1 translation by internal ribosome entry. Oncogene. 2000;19(7):899-905.

44. Cornelis S, Bruynooghe Y, Denecker G, Van Huffel S, Tinton S, Beyaert R. Identification and characterization of a novel cell cycle-regulated internal ribosome entry site. Mol Cell. 2000;5(4):597-605.

45. Creancier L, Mercier P, Prats AC, Morello D. C-myc internal ribosome entry site activity is developmentally controlled and subjected to a strong translational repression in adult transgenic mice. Mol Cell Biol. 2001;21(5): 1833-40.

46. Meyer N, Penn LZ. Reflecting on 25 years with MYC. Nat Rev Cancer. 2008; 8(12):976-90.

47. Evans JR, Mitchell SA, Spriggs KA, Ostrowski J, Bomsztyk K, Ostarek D, et al. Members of the poly $(\mathrm{rC})$ binding protein family stimulate the activity of the c-myc internal ribosome entry segment in vitro and in vivo. Oncogene. 2003;22(39):8012-20

48. Dormoy-Raclet V, Markovits J, Jacquemin-Sablon A, Jacquemin-Sablon H. Regulation of Unr expression by 5'- and 3'-untranslated regions of its mRNA through modulation of stability and IRES mediated translation. RNA Biol. 2005;2(3):e27-35.

49. Ivanov A, Mikhailova T, Eliseev B, Yeramala L, Sokolova E, Susorov D, et al. PABP enhances release factor recruitment and stop codon recognition during translation termination. Nucleic Acids Res. 2016;44(16):7766-76.

50. Smith RWP, Anderson RC, Larralde O, Smith JWS, Gorgoni B, Richardson WA, et al. Viral and cellular mRNA-specific activators harness PABP and elF4G to promote translation initiation downstream of cap binding. Proc Natl Acad Sci U S A. 2017;114(24):6310-5.

51. Patel GP, Ma S, Bag J. The autoregulatory translational control element of poly(a)-binding protein mRNA forms a heteromeric ribonucleoprotein complex. Nucleic Acids Res. 2005;33(22):7074-89.

52. Ray $S$, Anderson EC. Stimulation of translation by human Unr requires cold shock domains 2 and 4, and correlates with poly(a) binding protein interaction. Sci Rep. 2016;6:22461.

53. de Melo Neto OP, Standart N. Martins de Sa C. autoregulation of poly(a)binding protein synthesis in vitro. Nucleic Acids Res. 1995;23(12):2198-205.

54. Patel GP, Bag J. IMP1 interacts with poly(a)-binding protein (PABP) and the autoregulatory translational control element of PABP-mRNA through the $\mathrm{KH}$ III-IV domain. FEBS J. 2006;273(24):5678-90.

55. Cheng B, Kuppanda N, Aldrich JC, Akbari OS, Ferree PM. Male-killing Spiroplasma alters behavior of the dosage compensation complex during Drosophila melanogaster embryogenesis. Curr Biol. 2016;26(10):1339-45.

56. Kuroda Ml, Hilfiker A, Lucchesi JC. Dosage compensation in Drosophila-a model for the coordinate regulation of transcription. Genetics. 2016;204(2):435-50.

57. Moallem E, Kilav R, Silver J, Naveh-Many T. RNA-protein binding and posttranscriptional regulation of parathyroid hormone gene expression by calcium and phosphate. J Biol Chem. 1998;273(9):5253-9.

58. Dinur M, Kilav R, Sela-Brown A, Jacquemin-Sablon H, Naveh-Many T. In vitro evidence that upstream of $\mathrm{N}$-ras participates in the regulation of parathyroid hormone messenger ribonucleic acid stability. Mol Endocrinol (Baltimore, Md). 2006;20(7):1652-60

59. Osman AA, Neskey DM, Katsonis P, Patel AA, Ward AM, Hsu TK, et al. Evolutionary action score of TP53 coding variants is predictive of platinum response in head and neck Cancer patients. Cancer Res. 2015;75(7):1205-15.

60. Parker $\mathrm{R}$, Song $\mathrm{H}$. The enzymes and control of eukaryotic mRNA turnover. Nat Struct Mol Biol. 2004;11(2):121-7.
61. Chang TC, Yamashita A, Chen CY, Yamashita Y, Zhu W, Durdan S, et al. UNR, a new partner of poly(a)-binding protein, plays a key role in translationally coupled mRNA turnover mediated by the c-fos major coding-region determinant. Genes Dev. 2004;18(16):2010-23.

62. Grosset C, Chen CY, Xu N, Sonenberg N, Jacquemin-Sablon H, Shyu AB. A mechanism for translationally coupled mRNA turnover: interaction between the poly(a) tail and a c-fos RNA coding determinant via a protein complex. Cell. 2000;103(1):29-40.

63. Booy EP, McRae EK, Koul A, Lin F, McKenna SA. The long non-coding RNA $\mathrm{BC200}$ (BCYRN1) is critical for cancer cell survival and proliferation. Mol Cancer. 2017;16(1):109.

64. Booy EP, McRae EKS, Ezzati P, Choi T, Gussakovsky D, McKenna SA. Comprehensive analysis of the $\mathrm{BC} 200$ ribonucleoprotein reveals a reciprocal regulatory function with CSDE1/UNR. Nucleic Acids Res. 2018;46(21):11575-91.

65. Ju Lee H, Bartsch D, Xiao C, Guerrero S, Ahuja G, Schindler C, et al. A posttranscriptional program coordinated by CSDE1 prevents intrinsic neural differentiation of human embryonic stem cells. Nat Commun. 2017:8(1):1456.

66. Hunt SL, Hsuan JJ, Totty N, Jackson RJ. Unr, a cellular cytoplasmic RNA-binding protein with five cold-shock domains, is required for internal initiation of translation of human rhinovirus RNA. Genes Dev. 1999;13(4):437-48.

67. Grimmler M, Otter S, Peter C, Muller F, Chari A, Fischer U. Unrip, a factor implicated in cap-independent translation, associates with the cytosolic SMN complex and influences its intracellular localization. Hum Mol Genet. 2005;14(20):3099-111.

68. Moore KS, Yagci N, van Alphen F, Meijer AB, PAC t H, von Lindern M. Strap associates with Csde1 and affects expression of select Csde1-bound transcripts. PloS one. 2018;13(8):e0201690.

69. Duncan KE, Strein C, Hentze MW. The SXL-UNR corepressor complex uses a PABP-mediated mechanism to inhibit ribosome recruitment to msl-2 mRNA. Mol Cell. 2009;36(4):571-82.

70. Hennig J, Militti C, Popowicz GM, Wang I, Sonntag M, Geerlof A, et al. Structural basis for the assembly of the SxI-Unr translation regulatory complex. Nature. 2014;515(7526):287-90.

71. Duncan K, Grskovic M, Strein C, Beckmann K, Niggeweg R, Abaza I, et al. Sex-lethal imparts a sex-specific function to UNR by recruiting it to the msl2 mRNA 3' UTR: translational repression for dosage compensation. Genes Dev. 2006;20(3):368-79.

72. Gebauer F, Preiss T, Hentze MW. From cis-regulatory elements to complex RNPs and back. Cold Spring Harb Perspect Biol. 2012;4(7):a012245.

73. Aslam A, Mittal S, Koch F, Andrau JC, Winkler GS. The Ccr4-NOT deadenylase subunits CNOT7 and CNOT8 have overlapping roles and modulate cell proliferation. Mol Biol Cell. 2009;20(17):3840-50.

74. Coller J, Parker R. Eukaryotic mRNA decapping. Annu Rev Biochem. 2004;73: 861-90.

75. Goldstrohm AC, Hook BA, Seay DJ, Wickens M. PUF proteins bind Pop2p to regulate messenger RNAs. Nat Struct Mol Biol. 2006;13(6):533-9.

76. Sgromo A, Raisch T, Bawankar P, Bhandari D, Chen Y, Kuzuoglu-Ozturk D, et al. A CAF40-binding motif facilitates recruitment of the CCR4-NOT complex to mRNAs targeted by Drosophila Roquin. Nat Commun. 2017:8:14307.

77. Collier B, Gorgoni B, Loveridge C, Cooke HJ, Gray NK. The DAZL family proteins are PABP-binding proteins that regulate translation in germ cells. EMBO J. 2005;24(14):2656-66.

78. Graindorge A, Militti C, Gebauer F. Posttranscriptional control of X-chromosome dosage compensation. Wiley Interdiscip Rev RNA. 2011;2(4):534-45.

79. Militti C, Maenner S, Becker PB, Gebauer F. UNR facilitates the interaction of MLE with the IncRNA roX2 during Drosophila dosage compensation. Nat Commun. 2014;5:4762.

80. Martinez-Useros J, Garcia-Carbonero N, Li W, Fernandez-Acenero MJ, Cristobal I, Rincon R, et al. UNR/CSDE1 Expression Is Critical to Maintain Invasive Phenotype of Colorectal Cancer through Regulation of c-MYC and Epithelial-to-Mesenchymal Transition. J Clin Med. 2019;8(4):560.

81. Xie M, Zhou L, Chen X, Gainey LO, Xiao J, Nanes MS, et al. Progesterone and Src family inhibitor PP1 synergistically inhibit cell migration and invasion of human basal phenotype breast cancer cells. Biomed Res Int. 2015;2015:426429.

82. Crona J, Taieb D, Pacak K. New perspectives on Pheochromocytoma and Paraganglioma: toward a molecular classification. Endocr Rev. 2017;38(6): 489-515.

83. Liu P, Li M, Guan X, Yu A, Xiao Q, Wang C, et al. Clinical syndromes and genetic screening strategies of Pheochromocytoma and Paraganglioma. J Kidney Cancer VHL. 2018;5(4):14-22. 
84. Malik UU, Zarina S, Pennington SR. Oral squamous cell carcinoma: key clinical questions, biomarker discovery, and the role of proteomics. Arch Oral Biol. 2016;63:53-65.

85. You J, Lin L, Liu Q, Zhu T, Xia K, Su T. The correlation between the expression of differentiated embryo-chondrocyte expressed gene I and oral squamous cell carcinoma. Eur J Med Res. 2014;19:21.

86. Miller DS, Blessing JA, Krasner CN, Mannel RS, Hanjani P, Pearl ML, et al. Phase II evaluation of pemetrexed in the treatment of recurrent or persistent platinumresistant ovarian or primary peritoneal carcinoma: a study of the gynecologic oncology group. J Clin Oncol. 2009;27(16):2686-91.

87. Yin JY, Shen J, Dong ZZ, Huang Q, Zhong MZ, Feng DY, et al. Effect of elF3a on response of lung cancer patients to platinum-based chemotherapy by regulating DNA repair. Clin Cancer Res. 2011;17(13):4600-9.

88. Zhang Y, YU JJ, Tian Y, Li ZZ, Zhang CY, Zhang SF, et al. elF3a improve cisplatin sensitivity in ovarian cancer by regulating XPC and p27Kip1 translation. Oncotarget. 2015;6(28):25441-51.

89. van Dooijeweert B, van Ommen CH, Smiers FJ, Tamminga RYJ, Te Loo MW, Donker AE, et al. Pediatric diamond-Blackfan anemia in the Netherlands: an overview of clinical characteristics and underlying molecular defects. Eur J Haematol. 2018;100(2):163-70.

90. Guang S, Pang N, Deng X, Yang L, He F, Wu L, et al. Synaptopathology involved in autism Spectrum disorder. Front Cell Neurosci. 2018:12:470.

91. Bishop SL, Farmer C, Bal V, Robinson EB, Willsey AJ, Werling DM, et al. Identification of developmental and behavioral markers associated with genetic abnormalities in autism Spectrum disorder. Am J Psychiatry. 2017; 174(6):576-85.

92. Sanders SJ, Murtha MT, Gupta AR, Murdoch JD, Raubeson MJ, Willsey AJ, et al. De novo mutations revealed by whole-exome sequencing are strongly associated with autism. Nature. 2012;485(7397):237-41.

93. Lee AS, Kranzusch PJ, Doudna JA, Cate JH. elF3d is an mRNA cap-binding protein that is required for specialized translation initiation. Nature. 2016; 536(7614):96-9.

94. Chung H, Calis JJA, Wu X, Sun T, Yu Y, Sarbanes SL, et al. Human ADAR1 prevents endogenous RNA from triggering translational shutdown. Cell. 2018:172(4):811-24 e14.

95. Yin JY, Zhang JT, Zhang W, Zhou HH, Liu ZQ. elF3a: a new anticancer drug target in the elF family. Cancer Lett. 2018:412:81-7.

\section{Publisher's Note}

Springer Nature remains neutral with regard to jurisdictional claims in published maps and institutional affiliations.

Ready to submit your research? Choose BMC and benefit from:

- fast, convenient online submission

- thorough peer review by experienced researchers in your field

- rapid publication on acceptance

- support for research data, including large and complex data types

- gold Open Access which fosters wider collaboration and increased citations

- maximum visibility for your research: over $100 \mathrm{M}$ website views per year

At $\mathrm{BMC}$, research is always in progress.

Learn more biomedcentral.com/submissions 